\title{
RAPPRESENTAZIONI DELL'IDENTITÀ PROFESSIONALE DEGLI INSEGNANTI IN UN CONTESTO REGIONALE ITALIANO
}

\author{
REPRESENTATIONS OF THE PROFESSIONAL IDENTITY OF \\ EDUCATORS IN AN ITALIAN REGIONAL CONTEXT
}

\author{
Roberto Bonetto*
}

\begin{abstract}
Riassunto
II presente contributo è parte di una più ampia ricerca che si pone, come obiettivo principale, di indagare se vi siano delle conflittualità, delle discrepanze nelle differenti rappresentazioni di identità professionali degli insegnanti (ideale, reale, percepita) oltre a rintracciare e descrivere quei nuclei figurativi che costituiscono tali rappresentazioni sociali ovvero quali ne siano gli elementi centrali, più stabili e resistenti al cambiamento secondo quanto proposto dall'approccio strutturale (ABRIC, 1994, 1998). Lo spunto iniziale alla ricerca proviene da una ricerca condotta da di Lodolo D'Oria e collaboratori i quali considerano la conflittualità e la discrepanza della rappresentazione del Sé professionale degli insegnanti italiani, diviso tra ideale e reale, tra professionale e impiegatizio, la principale fonte di innesco di stati emozionali negativi, di sofferenza, disagio. Per contro, l'armonia tra le due tipologie del Sé (ideale e reale) e l'equilibrio tra identità personale e sociale promuovono benessere (LODOLO D'ORIA; POCATERRA; POZZI, 2003, p. 3).
\end{abstract}

Paroli chiavi: Rappresentazione sociale. Benessere. Malessere.

\begin{abstract}
This paper is part of a larger research intended to question whether there are conflicts and discrepancies in different representations of the professional identity of educators (ideal, real, "noticed"), and to describe the figurative nuclei that form such social representations, or what elements are central, more stable and resistant to change, according to the structural approach (ABRIC, 1994, 1998). The starting point of this study is a research conducted by Lodolo D'Oria et al. (2002), who consider the conflict and discrepancy in the representation of the professional Self of Italian educators (divided into ideal and real, professionals and employees) the main source of negative emotions, of suffering and gloom. On the other hand, the harmony between the two Self typologies (ideal and real) and the balance between social and personal identities provide well-being (D'ORIA; POCATERRA; POZZI, 2003).
\end{abstract}

Keywords: Social representations. Well-being. III-being.

\section{Introduzione}

I risultati della ricerca Getsemani, condotta su 3049 casi clinici di richiesta di inabilità al lavoro nella pubblica amministrazione italiana (LODOLO D'ORIA et al., 2002), rivelano che l'incidenza della sindrome da burnout tra gli insegnanti è 2-3 volte maggiore rispetto a quella di altre categorie di dipendenti pubblici (medici, infermieri, pubblici impiegati) ed è in costante e significativo aumento (dal $45 \%$ del biennio $92 / 93$ al $57,5 \%$ del biennio 2001/2). Nella stessa ricerca si ipotizza che il "disagio psichiatrico" si sviluppi da condizioni di prolungato stress lavorativo, che spesso porta appunto allo sviluppo del vero e proprio burnout, in accordo con quanto già sostenuto da Kyriacou (1987).

Ad una conclusione simile sono giunti anche Michael Huberman e Roland Vandemberge (1999)

\footnotetext{
Dottore di ricerca in Psicologia Sociale e della Personalità Università di Padova. E-mail: roberto.bonetto@istruzione.it
}

che hanno raccolto nel loro volume numerosi contributi e ricerche empiriche condotte sia in Nord America che in Europa. Molti degli studi citati in questa "agenda di ricerca" confermano che il burnout è largamente considerata una conclamata social issue (per una rassegna completa sull'argomento si veda Santinello e Negrisolo, 2009) e che gli insegnanti sono soggetti particolarmente a rischio (cfr. HUBERMANN; VANDEMBERGHE, 1999, cap. 1,2 ), ma altri studi, in apparente contraddizione, sembrano invece indicare che la maggior parte dei docenti non sperimenti particolari livelli di stress 0 di burnout (cfr. HUBERMANN; VANDEMBERGHE, 1999, cap. 8). Uno dei fattori "stressogeni", comuni a tutti i contesti considerati nelle ricerche riportate dai curatori del volume citato risiede ad un livello macro strutturale, ovvero nel profondo processo di revisione e riforma dei sistemi educativi nazionali che ha avuto e sta avendo luogo in questi ultimi anni praticamente in ogni paese industrializzato (cfr. HUBERMANN; VANDEMBERGHE, 1999, cap. 3). A questo "processo globale" di riforma fa eco una 
significativa trasformazione dei valori, della cultura professionale e delle pratiche degli insegnanti verso un'emergente "nuovo professionalismo": si assiste dunque al passaggio da una cultura dell'individualismo ad una della collaborazione, da un'organizzazione gerarchica al lavoro in team, dal controllo (supervisione) al supporto (mentoring/ tutoring), dal valore della formazione in servizio al valore dello sviluppo professionale, dall'autorità istituzionale dell'insegnante al contratto educativo con i genitori (HARGREAVES, 1994).

Vi è dunque una "pressione dall'alto" che spinge gli insegnanti verso un processo di sviluppo, di evoluzione, di ripensamento del loro ruolo professionale e delle pratiche ad esso connesse, ripensamento che "not only affects teachers' way of coping with occupational demands but also their understandings and representations of schools and teaching" (KELCHTERMANS, 1999, p. 183).

Questo sviluppo professionale (o evoluzione) può avere dunque un impatto anche riguardo quell'identità professionale, quel senso di sé o meglio quella rappresentazione sociale dell'identità professionale che gli insegnanti raggiungono, mantengono e sviluppano (o perdono) durante la loro vita lavorativa e che è di vitale importanza per comprendere le azioni e i coinvolgimenti degli stessi nel loro ambiente lavorativo (NIAS, 1993).

In questa sede riteniamo interessante sottolineare l'affermazione di Lodolo D'Oria e collaboratori che considera la conflittualità e la discrepanza della rappresentazione della propria professionalità, del proprio Sé professionale, diviso tra ideale e reale, tra professionale e impiegatizio, la principale fonte di innesco di stati emozionali negativi, di sofferenza, disagio, burnout. Per contro, l'armonia tra le due tipologie del Sé (ideale e reale) e l'equilibrio tra identità personale e sociale promuovono benessere. Ma uno degli aspetti più evidenziati dalla ricerca "[...] è l'emersione di un Sé professionale ideale legato alla figura di professionista, alla quale sono legate tutte le connotazioni di tipo positivo circa la propria realizzazione" (LODOLO D'ORIA; POCATERRA; POZZI, 2003, p. 3).

L'emersione del "professionista" a cui fanno riferimento Lodolo D'oria e colleghi trova almeno parziale sostegno in vari documenti ufficiali, sia italiani che internazionali. Ad esempio, nella ricerca promossa dall'OCSE (2006) si enfatizza la necessità di una professionalizzazione del ruolo docente centrata sul ripensamento della formazione, di base e in itinere degli insegnanti, che sposti l'asse d'intervento sull'acquisizione di competenze "tecniche" (didattiche, docimologiche, scientifiche, di ricerca ecc.) e non tanto (o non solo) di conoscenze. In ambito italiano, nell'introduzione del "Quaderno bianco sulla scuola" si dichiara: "La consapevolezza del ruolo strategico dell'istruzione per la crescita della persona, per la sua realizzazione e per lo sviluppo civile, democratico ed economico dell'Italia è cresciuta negli ultimi anni. Rafforzare la nostra dotazione di capitale materiale e immateriale è condizione indispensabile per tornare su un sentiero di sviluppo. II miglioramento della qualità della scuola, valorizzando la funzione e la dignità dell'insegnare, ne costituisce una priorità: può accrescere una mobilità sociale inadeguata, sospingere la produttività che ristagna, consolidare e diffondere il godimento pieno dei diritti di cittadinanza" (MIUR, 2007, p. i). All'interno, il documento propone a tal fine come prioritaria un'azione centrata, oltre che su azione di programmazione e di gestione delle politiche di investimento e valorizzazione delle risorse, sulla necessità di istituire un sistema di valutazione nazionale della qualità della scuola che favorisca l'identificazione di quelle criticità ormai conclamate del sistema nazionale educativo: "la diagnosi valutativa può divenire uno strumento importante di orientamento di ogni scuola nel disegnare un percorso formativo [degli insegnanti] che tenga conto degli effettivi bisogni [istituzionali]. [...] la formazione [degli insegnanti] diverrebbe così interesse di tutte le parti. In particolare, la necessità oggi avvertita di innovare i metodi di insegnamento, con particolare riferimento alle discipline tecnico-scientifiche e al metodo scientifico-sperimentale, si manifesterebbe attraverso le diagnosi e il confronto, scuola per scuola, e potrebbe effettivamente suscitare un salto di qualità nei processi formativi" (MIUR, 2007, p. XVIII).

Vi è, in buona sostanza, una crescente richiesta "istituzionale" di professionalizzazione dei docenti, accompagnata da marcati segnali di criticità dell'identità professionale degli stessi: "Risulta infatti che tutti i punti di riferimento tradizionali degli insegnanti si declinino oggi secondo la categoria della criticità. Gli interrogativi circa il senso dell'educare, da un lato, e la frammentazione del sapere, dall'altro, collocano oggi gli insegnanti in una posizione incerta e non più sostenuta dal sistema scuola, a sua volta in crisi" (LISIMBERTI, 2006, p. 29-30).

La risposta all'interrogativo di senso trova voce ufficiale nello slogan "Educare Istruendo" introdotto dall'allora ministro Fioroni nella premessa alle nuove indicazioni per il curricolo della scuola dell'infanzia e del primo ciclo di istruzione:

Obiettivo della scuola è quello di far nascere 'il tarlo' della curiosità, lo stupore della conoscenza, la voglia di declinare il sapere con la fantasia, la creatività, l'ingegno, la pluralità delle applicazioni delle proprie capacità, abilità e competenze. Tradotto in termini semplici: ci ostiniamo a pensare a una scuola che non abbia come obiettivo solo l'essere in funzione della richiesta del mercato. Solo se non si rinuncia ad educare istruendo si può mettere veramente a frutto l'unicità e l'irripetibilità di ogni singolo individuo. Solo così ogni persona può 
essere protagonista e costruire il proprio futuro in modi plurali, diversi ed innovativi. Per raggiungere questi obiettivi resta centrale l'acquisizione della cultura scientifica così come la valorizzazione dell'istruzione tecnica e professionale, campi nei quali il nostro paese ha costruito le fondamenta del proprio sviluppo. (MIUR, 2007, p. 6).

Ciò che ci sembra emergere dai documenti ufficiali è che la figura del docente fa riferimento ad una pratica e ad un'identità professionale che, per stessa ammissione dello stesso citato Ministro, risulta essere piuttosto complessa:

Nonc'ènessuna sindrome diburnoutnell'insegnante che non sia figlia del difficile incrocio fra ciò che dovremmo saper essere e saper fare e la straordinaria complessità che richiede l'educare istruendo proprio quella persona lì che, nella propria unicità, dà la misura della complessità dell'intrapresa e dell'ineludibilità del limite del nostro operare. Questa è la sfida. È questo il rischio educativo che gli insegnanti assumono nell'esercizio della propria professionalità. (MIUR, 2007, p. 7).

\section{L'identità professionale degli insegnanti in prospettiva psicosociale}

Riprendendo quanto precedentemente delineato, i più recenti studi sul burnout si sono sviluppati all'interno di un contesto teorico che pone in rilievo l'importanza dell'interazione e della relazione di bilanciamento/sbilanciamento tra risorse individuali e richieste ambientali-organizzative, tra armonia o disarmonia del proprio Sé professionale, ideale e reale.

II rischio che gli insegnanti assumono sembra dunque risiedere nella complessità e nella difficoltà del loro operare, nella difficoltà di reperire un senso e un'identità professionale al loro mestiere.

Risulta essenziale chiarire a questo punto a cosa ci si riferisce parlando di identità professionale $e$ come questa venga definita, con specifico riferimento all'identità professionale degli insegnanti, anche in altri ambiti accademici.

Per quanto riguarda il concetto di identità professionale degli insegnanti, riteniamo opportuno fare qui riferimento alle definizioni più recenti offerte dalla produzione scientifica pedagogica, sociologica e psico-sociale. Numerosi sono i tentativi di definizione dell'identità professionale docente che si possono incontrare in letteratura; alcuni più preoccupati di mostrarne i tratti caratteristici attraverso l'accostamento ad altre figure, altri più preoccupati di individuarne le componenti teoricoconcettuali. Tra i primi citiamo, a titolo esemplificativo, il modello "storico" proposto da Porcheddu (1978) che delinea alcuni profili di insegnanti susseguitesi nel corso dei decenni a partire dal profilo tradizionale dell'insegnante missionario di fine ottocento, oppure la figura proposta da Durkheim (1971) che invece fa riferimento all'insegnante sacerdote, trasmettitore di capacità, nozioni e valori. Più recenti profili di insegnanti sono stati raccolti in sette categorie: l'insegnante missionario tradizionale, lo specializzato, il tecnologico, il ricercatore, l'artista, l'interattivo e il guru (CHIOSSO; MARIANI, 2002). Tra i riferimenti che tendono invece a sottolinearne gli aspetti teorico-concettuali, riteniamo utile ai nostri fini riproporre la tripartizione avanzata dalla già citata ricerca OCSE (2006): l'insegnate professionista (del sociale e tecnico), l'artista, il funzionario pubblico/ impiegato. Di estrema utilità risultano, inoltre, le riflessioni offerte da Cristina Lisimberti (2006), la quale sintetizza la questione della definizione della professione e della professionalità docente attraverso uno schema riassuntivo che interseca "vari livelli di indagine epistemologica e disciplinare, differenti approcci e prospettive di studio" (LISIMBERTI, 2006, p. 67-68). L'autrice avanza la proposta di indagare professione-professionalità degli insegnanti attraverso l'intersezione tra due assi concettuali, ideale-reale e individuale-collettivo, che permetterebbero di focalizzarsi su specifici aspetti ora più legati alla professione concreta, individuale o collettiva, ora sugli aspetti di rappresentazione dell'insegnante orientati verso il dover essere individuale (reali/normativi) o verso l'ideale (collettivi). Come avremo meglio modo di dire in seguito, in questa sede privilegeremo questi ultimi aspetti.

Per quanto riguarda i riferimenti prodotti dalla psicologia sociale, ai fini del presente contributo considereremo i concetti di identità sociale e di sentimento di identità come punti di riferimento importanti. La prima viene definita da Tajfel come "quella parte dell'immagine di sé di un individuo che deriva dalla sua consapevolezza di appartenere ad un gruppo sociale (o più gruppi), unita al valore e al significato emotivo attribuito a tale appartenenza" (TAJFEL, 1995, p. 314). Per quanto riguarda il sentimento di identità, riteniamo illuminante la sintesi concettuale che Palmonari propone: "una qualità relazionale e temporale della rappresentazione di sé" (PALMONARI; CAVAZZA; RUBINI, 2002, p. 140). I due concetti non sono sovrapponibili, in quanto il primo si riferisce, in buona sostanza, ad un'identità definita in base a criteri oggettivi, "prodotta" da specifiche strutture sociali, ovvero identità tipiche (cfr. BERGER; LUCKMANN, 1969), mentre il secondo fa riferimento all'esperienza che l'attore sociale ha "di continuità nel tempo e nello spazio del proprio Sé". Vi è tuttavia "una stretta interdipendenza fra sentimento di identità e la nozione di identità definita in termini oggettivi dal momento che l'attore conosce quasi sempre come è definito socialmente e utilizza tale informazione come uno dei componenti del proprio 
sentimento di identità" (PALMONARI; CAVAZZA; RUBINI, 2002, p. 242). Avendo chiara questa distinzione, Palmonari offre una sintesi riguardo il rapporto tra identità sociale e identità personale, da questi inteso come

[...] due punti estremi di un continuum lungo il quale l'individuo, con il suo patrimonio evolutivo, nel suo ambiente sociale e nella sua storia, 'sente' la propria identità. Ad un estremo, quello sociale, ... in cui [l'individuo] è coinvolto a vivere un rapporto molto stretto con gli altri membri del proprio gruppo. Si sente interdipendente con tale gruppo e il suo impegno nell'azione sarà in rapporto con tale sentimento, il che influenza gli obiettivi che si pone, le strategie che sceglie, i simboli che utilizza, la lotta che sostiene in quanto 'identificato' con il gruppo. ... All'altro estremo, quello personale, l'individuo riflette sulla propria storia, sulla propria prospettiva temporale, in cui situa ricordi di varie connotazioni affettive, attese, speranze, i propri progetti, 'come se' fosse isolato. (PALMONARI; CAVAZZA; RUBINI, 2002, p. 243).

D'altronde già Tajfel sottolineava l'importanza di distinguere il comportamento intergruppi da quello interpersonale e, allo stesso, tempo collocava il comportamento sociale lungo un continuum ai cui estremi troviamo appunto i due "tipi di comportamento": "Nel primo caso, l'interazione è considerata determinata dall'appartenenza ai vari gruppi e dalle relazioni tra di loro; nel secondo, dipende in misura superiore dagli individui, dalle caratteristiche personali e dalle relazioni interpersonali." (BROWN, 2000, p. 19).

I richiami sopra menzionati a considerare il "continuum" tra due poli opposti, siano essi identità (sociale e personale) o comportamento (interpersonale e intergruppi), risultano particolarmente utili a ricordarci da una parte che sembra esistere una stretta connessione ed una continuità di significati tra identità e concetti di Sé (cfr. CARUGATI, 1979), dall'altra ad evitare riduzionismi che rischiano di estremizzare quelle differenze del Sé che soggiacciono e trovano invece unitarietà grazie alla funzione sintetizzante dell'Ego (ERICKSON, 1974).

In altre parole, se da un lato possiamo concordare nell'affermare che "non c'è dunque da stupirsi se nell'insegnante, figura inserita nel più grande apparato amministrativo dello Stato, convivono in equilibrio instabile elementi [identità] di tipo impiegatizio ed elementi di tipo professionale" (CORRADINI, 1982, p. 153), dall'altro riteniamo che tale convivenza appartenga alla "normale" conquista di identità - non esclusivamente professionale - vista come il "risultato di uno di quei conflitti vitali che ritmano il ciclo di vita dalla nascita alla vecchiaia" (PALMONARI; CAVAZZA; RUBINI 2002, p. 137). Vorremmo insomma tentare di vedere se quel conflitto, quella discrepanza tra Sé ideale e reale, tra identità sociale e personale che Lodolo D'Oria ipotizza essere innesco di stati di disagio abbia davvero stretti legami con il malessere o, nella sua versione "armonica", con il benessere degli insegnanti.

A questo scopo riteniamo utile un approccio che permetta di conservare una visione su entrambi gli aspetti fin qui richiamati, cioè sia quello individuale/interpersonale, sia quello sociale/ intergruppi, con una più marcata focalizzazione nei confronti di quest'ultimo aspetto. È per questa ragione che in questo studio abbiamo scelto di adottare la prospettiva delle rappresentazioni sociali (MOSCOVICl, 1961/1976) quale background teoretico e metodologico.

\section{Identità e rappresentazione sociale}

Palmonari (1981, p. 44), nella premessa alla ricerca da lui condotta sull'identità professionale degli psicologi, afferma che:

\begin{abstract}
Le rappresentazioni sociali hanno un'esistenza reale, dello stesso ordine di quella del linguaggio, del denaro, degli stati psichici di benessere o di sofferenza e appaiono dotate di una propria consistenza, come prodotti dall'azione e dalla comunicazione umana. Spesso sono il risultato di un lavoro professionale. Pensiamo a quello che fanno i pedagogisti, gli animatori culturali, certi giornalisti e tutti quelli che adempiono al ruolo di presentare la scienza, la cultura, la tecnica, l'arte al grande pubblico. [Gli insegnanti] Creano, trasmettono, spesso senza saperlo, rappresentazioni sociali. ... È vero che sono gli individui a pensarle e a produrle, ma nel corso di scambi, di atti di cooperazione, non in modo isolato. Sono cioè realtà condivise. ... Sono sempre sdoppiate in due facce: quella dell'immagine e quella del significato, che si corrispondono reciprocamente. La rappresentazione sociale, in altre parole, fa corrispondere ad ogni immagine un senso e ad ogni senso una immagine. (PALMONARI, 1981, p. 44).
\end{abstract}

Quando l'immagine identitaria (e di conseguenza anche il senso) - nel nostro caso trattiamo dell'immagine/rappresentazione sociale professionale dell'insegnante - è messa in discussione, minacciata (è appunto il nostro caso), facilmente si innescano, come abbiamo già detto 
precedentemente, stati di disagio. II riferimento allora alla nozione di rappresentazione sociale viene qui assunto comemodalità diorganizzazionedeicontenuti e delle valutazioni che definiscono e presiedono l'agire sociale dell'individuo che può diventare quel costrutto di mediazione che consente di superare la dicotomia individuo/gruppo, individuando il terreno comune adatto per esplorare teoricamente ed empiricamente il concetto di indentità professionale nonchè le possibili relazioni con il malessere e/o il benessere dei soggetti che compongono la categoria professionale indagata, i docenti appunto. Le variabili di riferimento ed i contenuti significativi dell'approccio psico-sociale adottato rimandano alla problematica delle aspettative, delle motivazioni, dei modelli che organizzano la conoscenza, dei processi di rappresentazione della realtà sociale che presiedono l'agire individuale.

Infatti le rappresentazioni sociali sono elaborazione di un oggetto sociale da parte di una comunità che permette ai suoi membri di comportarsi, di agire e di comunicare in modo comprensibile, utili per la scoperta, l'organizzazione e la ricostruzione della realtà (MOSCOVICI, 1963). Prendiamo nuovamente a prestito un passaggio di Palmonari che chiarisce ulteriormente cosa sono e a cosa servono le rappresentazioni sociali: "Ci sono ovviamente, entro ogni società, accordi e conflitti, cooperazione e competizione, scontri di mentalità e sforzi di comprensione ma ogni gruppo attivo sulla scena sociale ha l'esigenza di mantenere il più possibile coerente l'insieme delle credenze e delle teorie di senso comune su cui la vita stessa del gruppo si fonda" (PALMONARI; CAVAZZA; RUBINI, 2002, p. 85).

II padre della teoria delle rappresentazioni sociali individua due processi fondamentali che danno origine alle stesse: l'ancoraggio e l'oggettivazione (MOSCOVICl, 1961/1976). II primo processo permette di includere qualcosa di non familiare, qualcosa che risulta problematico, inserendolo nella rete di concetti/categorie a noi familiari, all'interno di una cornice di riferimento (categoria) a noi ben nota al fine di potere interpretare e confrontare questo "oggetto" alla luce delle caratteristiche tipiche della categoria conosciuta. L'ancoraggio, in altre parole, permette "l'integrazione cognitiva (funzionale) dell'oggetto rappresentato nel sistema di pensiero preesistente" (JODELET, 1984, p. 371). Attraverso il processo di oggettivazione, strettamente connesso con l'ancoraggio, i concetti astratti, non familiari, le novità, gli "oggetti" che disturbano la nostra rappresentazione preesistente, vengono trasformati in immagini "concrete", percepibili e comunicabili. Il processo di oggettivazione permette quindi la costituzione di un "nucleo figurativo" che può essere inteso come il minimo comune denominatore che consente di presentare un "oggetto sociale" agli altri e a sè stessi in modo omogeneo.
Nel contributo che proponiamo dedicheremo ampio spazio al processo di oggettivazione e alla descrizione proprio di quel nucleo figurativo che costituisce la rappresentazione sociale dell'identità professionale degli insegnanti facendo riferimento principalmente all'approccio strutturale, proposto da Abric (1994, 1998). L'approccio strutturale, detto in estrema sintesi, è una prospettiva sperimentale la quale assume che, al fine di interpretare la realtà, le persone si riferiscano a rappresentazioni sociali o "schemi" che sono generati e condivisi all'interno dei gruppi di cui fanno parte. Essi hanno anche la funzione di mantenere l'identità sociale del gruppo e di fornire guida e giustificazione all'agire quotidiano riguardo social issues rilevanti (ABRIC, 1994). La teoria principale dell'approccio strutturale, detta del "nucleo centrale" ipotizza che che le rappresentazioni sociali siano organizzate da due sistemi differenti, ognuno con specifiche funzioni e caratteristiche: il sistema centrale e il sistema periferico. II primo racchiude relativamente pochi elementi i quali organizzano, strutturano la rapprresentazione e ne determinano il significato. Questi elementi sono quelli più stabili e resistenti al cambiamento. Tutti gli altri elementi della rappresentazione compongono invece il secondo sistema, ovvero quello periferico. Essi, oltre ad essere molto più numeorsi, dipendono dal nucleo centrale ed hanno la funzione di "adattare" la rappresentazione a particolari contesti di realtà, collegandola all'agire quotidiano (FLAMENT, 1994). II sistema periferico ha inoltre la funzione di "proteggere" il nucleo centrale dalle trasformazioni attraverso l'assimilazione e l'accomodamento dei cambiamenti stessi. Attraverso l'interazione dei due sistemi, una rappresentazione può essere allo stesso tempo rigida e stabile, da una parte, e flessibile dall'altra (ABRIC, 1994). Ai fini della presente ricerca ci proponiamo principalmente di individuare quali siano gli elementi centrali, ovvero quelli più stabili e resistenti al cambiamento, della rappresentazione dell'identità professionale degli insegnanti.

\section{Partecipanti, metodo e procedura di analisi}

Tra novembre e dicembre 2009 sono stati distribuiti circa 900 questionari ad insegnanti di scuola primaria (ex scuola elementare) e secondaria di II grado (ex scuola media) appartenenti a nove istituti comprensivi della provincia di Padova. Una parte del questionario richiedeva ai partecipanti di esprimere, attraverso libere associazioni di parole e/o brevi frasi, quali caratteristiche dovessero possedere tre tipologie di insegnanti: l'insegnante ideale, l'insegnante reale e l'insegnante percepito dall'utenza. Un esempio delle richieste è la seguente:

\footnotetext{
"Quali caratteristiche ritiene possieda un insegnante reale che opera quotidianamente nella scuola di
} 
oggi? Senza pensarci troppo, scriva nello spazio sottostante le prime quattro caratteristiche che le vengono in mente con parole-chiave o brevi affermazioni".

Successivamente si chiedeva di classificare le libere associazioni in ordine di importanza, dalla più alla meno importante. Dai 418 questionari restituiti, per la prima tipologia sono state ottenute 412 risposte utili (sei questionari non presentavano alcuna evocazione) per un totale di 1646 evocazioni e complessive 233 parole differenti. Per la seconda, le risposte utili sono state 393, 1557 le evocazioni complessive e 381 parole differenti mentre per la terza tipologia, l'insegnante "percepito", le risposte utili sono state 390 per un totale di 1445 evocazioni e 413 parole differenti. Coerentemente con la procedura di analisi proposta da Vergérs (1992), il programma EVOCATION 2000 permette di ottenere quattro quadranti in cui vengono raggruppate le parole secondo frequenza e rango di "evocazione". II "punto di divisione" di ciascun raggruppamento è stato scelto scegliendo un criterio il più possibile omogeneo. Innanzitutto, per tutte e tre le tipologie sono state escluse tutte le evocazioni con frequenza minore di 5 , mentre per la soglia intermedia, quella di divisione, si è scelto di considerare una frequenza il più possibile vicina al 50\%: per quanto riguarda l'insegnante ideale, la frequenza 31 , scelta come frequenza intermedia, rappresenta il $51.9 \%$ del totale delle evocazioni; per il "reale" la frequenza 13 è pari al $51.6 \%$ mentre per il "percepito" la frequenza 10 è pari al $52.9 \%$ del totale. Infine, anche per quanto riguarda l'ordine di evocazione è stato scelto il rango medio come punto di divisione.

Le evocazioni così suddivise sono state marcate con cinque simboli differenti corrispondenti ad altrettanti raggruppamenti categoriali: $(P)$ caratteristiche tecnico-professionali, (I) caratteristiche individuali/di personalità, (R) caratteristiche relazionali/comunicative ed infine (S) caratteristiche sociali. L'uso del segno meno ( - ) dopo ogni parola vuole mettere in evidenza caratteristiche comunemente considerate negative. Inoltre è stato condotto il test di centralità sulle evocazioni analizzate prendendo in considerazione l'evocazione considerata come più importante dai rispondenti. Vengono quindi confermate nel nucleo centrale della rappresentazione quelle evocazioni indicate come più importanti che ottengono una frequenza maggiore o uguale al $40 \%$ sulla frequenza totale della singola evocazione. I risultati ottenuti verranno descritti singolarmente.

\section{L'insegnante ideale}

Tabella 1 - Insegnante IDEALE

\begin{tabular}{|c|c|c|c|c|c|}
\hline \multicolumn{3}{|c|}{ Quadrante 1 - Probabili elementi del nucleo centrale } & \multicolumn{3}{|c|}{ Quadrante 2 - Elementi intermedi } \\
\hline & Frequenza $\geq 31$ & Rango medio $<2.5$ & & Frequenza $\geq 31$ & Rango medio $\geq 2.5$ \\
\hline $\begin{array}{l}\text { (I)autorevolezza } \\
\text { (R)capacità_relazionale* } \\
\text { (P)competenza* } \\
\text { (P)competenza_materia* } \\
\text { (I)empatia } \\
\text { (I)motivazione* } \\
\text { (I)passione* } \\
\text { (I)pazienza } \\
\text { (P)preparazione* } \\
\text { (P)professionalità* }\end{array}$ & $\begin{array}{c}54 \\
70 \\
100 \\
31 \\
44 \\
31 \\
47 \\
136 \\
99 \\
72\end{array}$ & $\begin{array}{l}2.315 \\
2.229 \\
2.080 \\
2.097 \\
2.341 \\
1.903 \\
2.404 \\
2.221 \\
1.909 \\
2.014\end{array}$ & $\begin{array}{l}\text { (R)capacità_comunicativa } \\
\text { (R)collaborativo } \\
\text { (I)creatività } \\
\text { (I)disponibilità } \\
\text { (I)flessibilità }\end{array}$ & $\begin{array}{l}47 \\
36 \\
31 \\
79 \\
39\end{array}$ & $\begin{array}{l}2.723 \\
3.250 \\
3.032 \\
2.696 \\
2.641\end{array}$ \\
\hline \multicolumn{3}{|c|}{ Quadrante 3 - Elementi intermedi } & \multicolumn{3}{|c|}{ Quadrante 4 - Elementi periferici } \\
\hline & Frequenza $<31$ & Rango medio $<2.5$ & & Frequenza $<31$ & Rango medio $\geq 2.5$ \\
\hline $\begin{array}{l}\text { (I)accoglienza } \\
\text { (I)adattabilità } \\
\text { (I)amore_alunni } \\
\text { (R)capacità_ascolto } \\
\text { (R)capacità_coinvolgere } \\
\text { (R)capacità_gestione_ } \\
\text { gruppo } \\
\text { (I)carisma } \\
\text { (I)chiarezza } \\
\text { (P)competenza_didattica } \\
\text { (P)conoscenza } \\
\text { (P)conoscenza_materia } \\
\text { (P)cultura } \\
\text { (I)dedizione } \\
\text { (R)disponibilità_ascolto } \\
\text { (I)educatore } \\
\text { (I)equilibrio } \\
\text { (I)serenità } \\
\text { (I)serietà } \\
\text { (I)umanità* }\end{array}$ & $\begin{array}{c}5 \\
6 \\
8 \\
24 \\
16 \\
5 \\
12 \\
17 \\
26 \\
9 \\
16 \\
22 \\
7 \\
6 \\
5 \\
11 \\
7 \\
6 \\
12\end{array}$ & $\begin{array}{l}2.400 \\
2.333 \\
2.125 \\
2.292 \\
2.375 \\
2.200 \\
2.417 \\
2.353 \\
2.385 \\
2.333 \\
1.938 \\
2.318 \\
2.286 \\
2.000 \\
1.800 \\
1.818 \\
2.286 \\
2.000 \\
2.417\end{array}$ & $\begin{array}{l}\text { (P)aggiornato } \\
\text { (I)amore_lavoro } \\
\text { (I)apertura_mentale } \\
\text { (I)attenzione } \\
\text { (I)autocontrollo } \\
\text { (I)coerenza } \\
\text { (R)comprensione } \\
\text { (P)conosc_psicopeda- } \\
\text { gogica } \\
\text { (R)diplomazia } \\
\text { (I)elasticità } \\
\text { (I)entusiasmo } \\
\text { (I)esperienza } \\
\text { (I)fermezza } \\
\text { (I)imparziale } \\
\text { (I)impegno } \\
\text { (I)Precisione } \\
\text { (I)Sensibilità } \\
\text { (I)severità } \\
\text { (I)simpatia } \\
\text { (I)tolleranza } \\
\text { (I)umiltà }\end{array}$ & $\begin{array}{c}21 \\
9 \\
14 \\
13 \\
10 \\
14 \\
23 \\
6 \\
6 \\
10 \\
20 \\
6 \\
10 \\
5 \\
8 \\
5 \\
16 \\
5 \\
17 \\
9 \\
10\end{array}$ & $\begin{array}{l}3.095 \\
3.111 \\
3.357 \\
2.615 \\
3.400 \\
2.643 \\
2.696 \\
2.833 \\
3.000 \\
3.000 \\
3.350 \\
2.667 \\
3.300 \\
3.200 \\
3.125 \\
3.200 \\
2.750 \\
3.400 \\
3.059 \\
2.256 \\
3.000\end{array}$ \\
\hline
\end{tabular}

${ }^{*}{ }^{*}$ Elemento confermato come appartenente al nucleo centrale. 
A partire dall'analisi lessicografica riportata in Tabella 1, il probabile nucleo centrale della rappresentazione sociale dell'insegnante ideale risulta composto da quattro caratteristiche che potremmodefiniretecnico-professionali(competenza, competenza della materia, preparazione e professionalità). da cinque caratteristiche individuali o di personalità (autorevolezza, empatia, motivazione, passione e pazienza) e una relazionale-comunicativa (capacità relazionale).

Risultano confermate centrali dal test di centralità le evocazioni competenza, competenza materia, capacità relazionale, motivazione, passione e professionalità.

Per completare l'analisi della probabile rappresentazione sociale dell'insegnante ideale, utilizzando il programma Evocation 2000 si è proceduto a creare, a partire dalla prossimità semantica con le evocazioni di maggior frequenza, 29 categorie differenti che includono 1373 evocazioni $(83.4 \%$ sul totale). Nel creare le categorie si è preferito lasciare distinte la categoria competenza (generica) da competenza didattica e competenza della materia in modo da non semplificare troppo specificità numericamente importanti, criterio poi seguito anche nelle successive analisi. Tale categorizzazione è stata quindi sottoposta ad analisi utilizzando il software Similitude 2000 il quale permette di visualizzare le connessioni tra gli elementi che partecipano alla composizione prototipica del nucleo centrale della rappresentazione sociale in esame attraverso la relazione di legame associativo (co-occorrenza) tra parole nella convinzione che quanto più un concetto è centrale, tanto più presenta legami con altri concetti (MOLINER, 1994).

Gráfico 1 - Rappresentazione dei legami (albero massimo) tra categorie di evocazioni per insegnante ideale

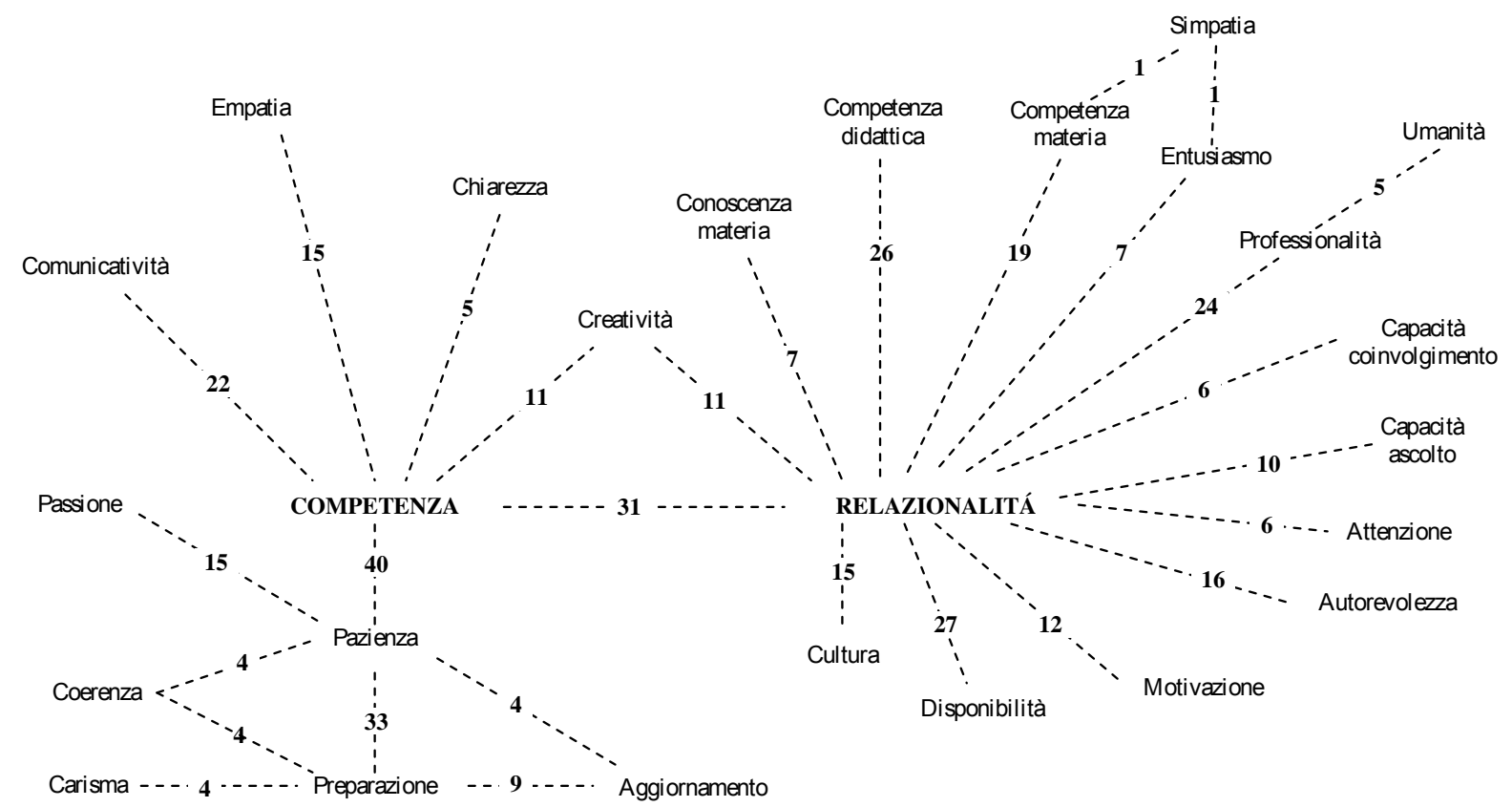

Come si può notare dal Grafico 1 si possono individuare due poli attorno ai quali si costruisce la rappresentazione professionale dell'insegnante ideale: relazionalità e competenza, entrambe caratteristiche appartenenti al nucleo centrale. La caratteristica relazionale risulta fortemente connessa sia a caratteristiche professionalirelazionali (competenza didattica, competenza materia, conoscenza materia, professionalità, cultura, capacità di ascolto, di coinvolgimento), sia a caratteristiche individuali o di personalità (disponibilità, autorevolezza, motivazione, entusiasmo, creatività, attenzione), mentre il secondo "polo" presenta quasi esclusivamente relazioni con caratteristiche individuali o di personalità (pazienza, empatia, chiarezza, creatività). Analizzando ulteriormente $\mathrm{i}$ legami che compongono la rete delle co-occorrenze, si possono individuare alcune relazioni interessanti. La prima può essere letta nella triangolazione tra competenza, relazionalità $e$ creatività: l'insegnante ideale possiede una capacità fondamentale, quella relazionale, non disgiunta da una certa dose di creatività, necessaria forse a rendere più personale, più umana e meno "artificiale", meno tecnicamente "fredda", la competenza. A confortare questa interpretazione sono le relazioni che legano competenza e pazienza ma anche la stessa pazienza legata a sua volta a passione, preparazione e aggiornamento. In entrambi i casi è ravvisabile un asse di connessione dei due termini "professionali" (competenza e preparazione) con caratteristiche o tratti di personalità (pazienza e 
passione), quasi ad indicare che le "doti umane" o personali siano tratti indispensabili al possesso o alla creazione di competenze professionali o, viceversa, che le competenze professionali necessitino, per essere "vere", di essere tradotte e/o mediate da specifici tratti di personalità. Questo aspetto viene, secondo noi, ulteriormente rinforzato dalle relazioni che competenza ha con empatia e con comunicatività: di nuovo un tratto di personalità (empatia) e uno comunicativo a testimonianza della concezione personale-relazionale della competenza ideale. Interessanti risultano essere anche i legami dell'aspetto relazionale, centrale e connesso con molte caratteristiche "professionali" da un lato (competenza didattica, competenza materia, cultura e professionalità) e tratti di personalità (flessibilità, motivazione e disponibilità), di nuovo a suggerire che la compresenza di aspetti "hard" e "soft" viene considerata una componente necessaria all'insegnante ideale. Abbastanza informativa, a nostro avviso, è anche la mancanza di connessioni "dirette" tra le caratteristiche professionali presenti nel grafico. Questo può significare che le caratteristiche professionali, comunque importanti, siano considerate come equivalenti e quindi in realtà facciano parte di una stessa categoria concettuale di base. Possiamo concludere affermando che nella rappresentazione dell'insegnante ideale concorrono due "aspetti centrali": il sapere, che è anche "saper fare" (competenza), e il "saper essere" (tratto relazionale-personale).

\section{L'insegnante reale}

Tabella 2 - Insegnante REALE

\begin{tabular}{|c|c|c|c|c|c|}
\hline \multicolumn{3}{|c|}{ Quadrante 1 - Probabili elementi del nucleo centrale } & \multicolumn{3}{|c|}{ Quadrante 2 - Elementi intermedi } \\
\hline & Frequenza $\geq 13$ & Rango medio $<2.5$ & & Frequenza $\geq 13$ & Rango medio $\geq 2.5$ \\
\hline $\begin{array}{l}\text { (I)adattabilità } \\
\text { (P)competenza* } \\
\text { (P)competenza_materia* } \\
\text { (P)competenza_didattica } \\
\text { (P)conoscenza_materia* } \\
\text { (I)demotivazione- } \\
\text { (I)impegnato } \\
\text { (I)flessibilità } \\
\text { (I)pazienza } \\
\text { (I)passione* } \\
\text { (P)preparazione } \\
\text { (P)professionalità } \\
\text { (I)stanchezza- } \\
\text { (I)stressato- }\end{array}$ & $\begin{array}{l}31 \\
86 \\
17 \\
13 \\
15 \\
28 \\
31 \\
43 \\
140 \\
17 \\
82 \\
54 \\
21 \\
16\end{array}$ & $\begin{array}{l}2.129 \\
2.035 \\
2.000 \\
1.692 \\
1.600 \\
2.286 \\
2.356 \\
2.280 \\
2.074 \\
2.471 \\
2.280 \\
2.074 \\
2.476 \\
2.250\end{array}$ & $\begin{array}{l}\text { (R)capacità relazionale } \\
\text { (I)disponibilità } \\
\text { (I)frustrazione- } \\
\text { (I)autorevolezza } \\
\text { (I)creatività } \\
\text { (I)elasticità } \\
\text { (I)motivazione } \\
\text { (S)responsabilità } \\
\text { (R)collaborativo }\end{array}$ & $\begin{array}{l}26 \\
66 \\
22 \\
16 \\
19 \\
13 \\
15 \\
14 \\
20\end{array}$ & $\begin{array}{l}2.615 \\
2.576 \\
2.636 \\
2.875 \\
2.778 \\
2.600 \\
2.800 \\
2.800 \\
2.950\end{array}$ \\
\hline \multicolumn{3}{|c|}{ Quadrante 3 - Elementi intermedi } & \multicolumn{3}{|c|}{ Quadrante 4 - Elementi periferici } \\
\hline & Frequenza $<13$ & Rango medio $<2.5$ & & Frequenza $<13$ & Rango medio $\geq 2.5$ \\
\hline $\begin{array}{l}\text { (I)ansioso- } \\
\text { (R)capac_gestione_ } \\
\text { gruppo } \\
\text { (P)conoscenza } \\
\text { (P)cultura } \\
\text { (I)diplomatico } \\
\text { (I)esperienza } \\
\text { (I)frettoloso- } \\
\text { (I)individualista- } \\
\text { (I)insoddisfazione- } \\
\text { (I)preoccupazione- } \\
\text { (I)rassegnazione- } \\
\text { (P)scarsa competenza- } \\
\text { (I)scarsa_motivazione- } \\
\text { (I)scarsa pazienza- } \\
\text { (I)scarso entusiasmo- } \\
\text { (I)senso_dovere } \\
\text { (I)serietà } \\
\text { (I)sfiduciato- }\end{array}$ & $\begin{array}{c}5 \\
6 \\
5 \\
12 \\
9 \\
12 \\
5 \\
7 \\
8 \\
8 \\
6 \\
7 \\
9 \\
5 \\
5 \\
6 \\
11 \\
6\end{array}$ & $\begin{array}{l}2.200 \\
2.333 \\
1.800 \\
2.167 \\
2.444 \\
1.833 \\
2.200 \\
2.286 \\
2.000 \\
2.250 \\
1.667 \\
2.286 \\
1.444 \\
2.400 \\
2.000 \\
2.333 \\
2.364 \\
2.333\end{array}$ & $\begin{array}{l}\text { (P)aggiornato } \\
\text { (I)apertura_mentale } \\
\text { (I)attenzione } \\
\text { (I)buona volontà } \\
\text { (R)capacità_ascolto } \\
\text { (R)capacità comunicativa } \\
\text { (R)capacità_mediazione } \\
\text { (P)capacità organizzative } \\
\text { (R)comprensione } \\
\text { (I)dedizione } \\
\text { (I)deluso } \\
\text { (I)difficoltà relazione } \\
\text { (I)entusiasmo } \\
\text { (I)mediatore } \\
\text { (I)rigidità- } \\
\text { (I)rispetto } \\
\text { (P)scarso aggiornamento } \\
\text { (I)severità } \\
\text { (I)tolleranza } \\
\text { (I)volontà }\end{array}$ & $\begin{array}{c}9 \\
7 \\
11 \\
9 \\
6 \\
12 \\
5 \\
9 \\
9 \\
6 \\
5 \\
5 \\
7 \\
5 \\
4 \\
8 \\
5 \\
5 \\
5 \\
11\end{array}$ & $\begin{array}{l}3.111 \\
3.000 \\
3.000 \\
2.556 \\
3.000 \\
2.750 \\
2.800 \\
2.556 \\
2.778 \\
2.500 \\
2.600 \\
2.600 \\
3.143 \\
3.214 \\
3.000 \\
3.600 \\
3.200 \\
2.800 \\
2.636 \\
3.333\end{array}$ \\
\hline
\end{tabular}

(*) Elemento confermato come appartenente al nucleo centrale (test centralità).

Comesipuòfacilmentenotarein Tabella2, anche nel probabile nucleo centrale della rappresentazione sociale dell'insegnante reale possiamo ritrovare quattro caratteristiche professionali ed una individuale (già incontrati nel nucleo dell'insegnante ideale): pazienza, professionalità, preparazione, competenza e competenza materia. Di queste, competenza e competenza della materia risultano essere confermate dal test di centralità, conferma che riguarda anche conoscenza materia e passione. Rispetto all'insegnante ideale è da notare che manca nel nucleo centrale la competenza 
relazionale e si può notare una prevalenza piuttosto accentuata di caratteristiche individuali rispetto a quelle professionali, tre delle quali (demotivazione, stanchezza e stressato) sono negative, indice di un maggior riferimento delle caratteristiche evocate all'esperienza e alla pratica quotidiana, vissuta come faticosa. La rappresentazione complessiva sembra quindi testimoniare la presenza di criticità reali legate sia al ruolo professionale, sia a tratti di personalità a testimonianza che la professione è percepita come logorante e poco soddisfacente (stanchezza, frustrazione, demotivazione).

Successivamente, si è proceduto a creare 27 categorie, comprendenti 1087 evocazioni (69.8\% sul totale). I grafici risultanti sono i seguenti:

Gráfico 2 - Rappresentazione dei legami (albero massimo) tra categorie delle evocazione per insegnante reale

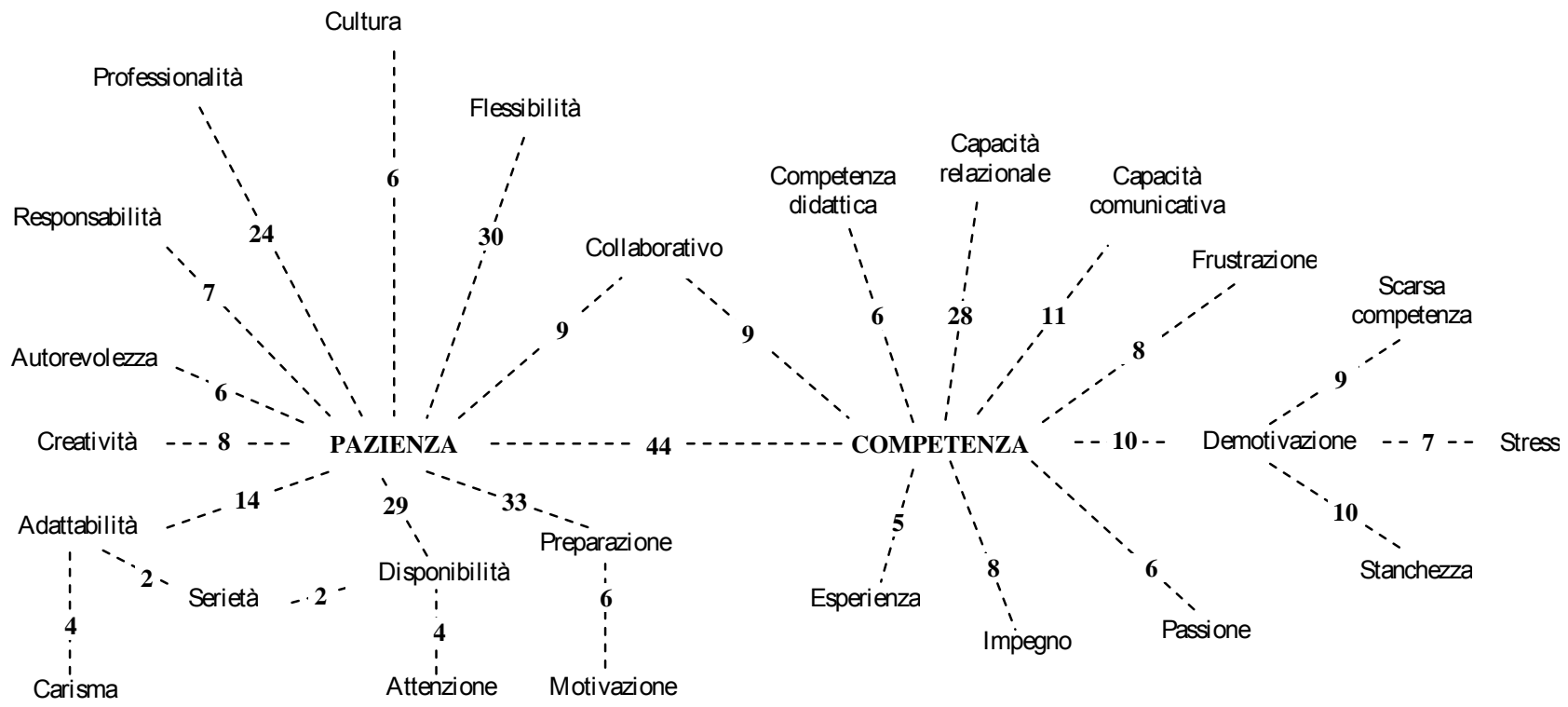

Notiamo subito come in questo caso la capacità relazionale abbia perso quella "centralità" che possedeva invece nella rappresentazione grafica dei legami tra caratteristiche evocate per l'insegnante ideale. Anche in questo caso troviamo due poli organizzatori: competenza e pazienza. La competenza presenta legami consistenti (quantitativamente) con capacità relazionale e comunicativa. Inoltre la triangolazione tra pazienza, competenza e disponibilità alla collaborazione (collaborativo) suggerisce da una parte che vi sia una specificità della competenza, genericamente intesa, che necessita non solo di doti umane (pazienza) ma anche di capacità di relazionarsi in maniera positiva con gli altri. Osservando le ramificazioni dirette della competenza, si può notare che i legami con le caratteristiche personali risultano deboli. Tra queste ultime spiccano caratteristiche personali "negative", quasi esclusivamente legate tra loro in un unico "ramo": la demotivazione. Pazienza mostra invece legami consistenti quantitativamente sia con doti personali (flessibilità, disponibilità, adattabilità) sia con caratteristiche professionali (professionalità e preparazione) confermando, a nostro avviso, l'importanza-centralità dell'asse personale-professionale della rappresentazione sociale dell'insegnante reale. Apparentemente dunque la rappresentazione sociale dell'insegnante reale sembra organizzata attorno ad un nucleo di caratteristiche personali-individuali collegate ad un saper fare professionale che sembra essere percepito come qualità individuale più che di categoria: infatti, la capacità relazionale sembra ricoprire un ruolo di presenza secondaria. Inoltre questa professionalità individuale viene presentata, anche se "marginalmente", come frustrata in quanto gli insegnanti, come categoria di "attori sociali di tutti i giorni", si percepiscono abbastanza demotivati, stanchi, stressati. 


\section{L’insegnante percepito}

Tabella 3 - Insegnante PERCEPITO

\begin{tabular}{|c|c|c|c|c|c|}
\hline \multicolumn{3}{|c|}{ Quadrante 1 - Probabili elementi del nucleo centrale } & \multicolumn{3}{|c|}{ Quadrante 2 - Elementi intermedi } \\
\hline & Frequenza $\geq 10$ & Rango medio $<2.5$ & & Frequenza $\geq 10$ & Rango medio $\geq 2.5$ \\
\hline $\begin{array}{l}\text { (P)competenza } \\
\text { (P)competenza_materia } \\
\text { (R)comprensione } \\
\text { (P)cultura } \\
\text { (I)disponibilità } \\
\text { (I)esigente } \\
\text { (S)fannullone- } \\
\text { (P)impreparazione- } \\
\text { (P)incompetenza- } \\
\text { (S)lavora poco- } \\
\text { (I)pazienza } \\
\text { (I)permissivo } \\
\text { (P)preparazione } \\
\text { (S)privilegiati- } \\
\text { (P)professionalità } \\
\text { (I)rigidità- } \\
\text { (I)scarsa motivazione- } \\
\text { (P)scarsa professionalità- } \\
\text { (I)tuttologo }\end{array}$ & $\begin{array}{l}67 \\
10 \\
24 \\
12 \\
72 \\
15 \\
13 \\
34 \\
24 \\
33 \\
91 \\
12 \\
74 \\
20 \\
43 \\
13 \\
10 \\
14 \\
13\end{array}$ & $\begin{array}{l}1.731 \\
1.700 \\
2.417 \\
1.583 \\
2.292 \\
2.333 \\
1.462 \\
1.971 \\
2.000 \\
2.061 \\
2.099 \\
2.471 \\
1.703 \\
2.200 \\
2.302 \\
2.308 \\
1.600 \\
1.357 \\
1.923\end{array}$ & $\begin{array}{l}\text { (I)autorevolezza } \\
\text { (R)capacità comuni- } \\
\text { cativa } \\
\text { (R)capacità relazionale } \\
\text { (P)incapacità- } \\
\text { (S)responsabilità } \\
\text { (I)scarsa comprensione } \\
\text { (I)scarsa disponibilità } \\
\text { (I)serietà } \\
\text { (I)severità }\end{array}$ & $\begin{array}{l}19 \\
11 \\
16 \\
11 \\
10 \\
15 \\
14 \\
14 \\
20\end{array}$ & $\begin{array}{l}2.579 \\
2.545 \\
3.063 \\
2.818 \\
3.100 \\
2.773 \\
2.733 \\
2.786 \\
2.500\end{array}$ \\
\hline \multicolumn{3}{|c|}{ Quadrante 3 - Elementi intermedi } & \multicolumn{3}{|c|}{ Quadrante 4 - Elementi periferici } \\
\hline & Frequenza $<10$ & Rango medio $<2.5$ & & Frequenza $<10$ & Rango medio $\geq 2.5$ \\
\hline $\begin{array}{l}\text { (R)accoglienza } \\
\text { (I)affidabilità } \\
\text { (I)arroganza } \\
\text { (S)babysitter } \\
\text { (R)capacità_ascolto } \\
\text { (P)competenza didattica } \\
\text { (P)conoscenza } \\
\text { (P)conoscenza_materia } \\
\text { (I)impegno } \\
\text { (I)risolutore problemi } \\
\text { (I)scarsa_pazienza- } \\
\text { (I)superficialità- } \\
\text { (I)troppo_severi- } \\
\text { (S)tempo_libero }\end{array}$ & $\begin{array}{l}5 \\
5 \\
5 \\
7 \\
9 \\
7 \\
9 \\
8 \\
9 \\
5 \\
6 \\
5 \\
5 \\
8\end{array}$ & $\begin{array}{l}1.800 \\
2.400 \\
2.400 \\
1.857 \\
1.889 \\
2.143 \\
1.778 \\
2.125 \\
2.222 \\
1.800 \\
1.500 \\
2.000 \\
2.400 \\
2.125\end{array}$ & $\begin{array}{l}\text { (P)aggiornato } \\
\text { (I)attenzione } \\
\text { (R)capacità_dialogo } \\
\text { (P)capacità_organiz- } \\
\text { zativa } \\
\text { (P)capacità_valutazione } \\
\text { (R)collaborativo } \\
\text { (I)creatività } \\
\text { (I)dedizione } \\
\text { (I)demotivazione } \\
\text { (I)esperienza } \\
\text { (I)flessibilità } \\
\text { (I)ignoranza- } \\
\text { (I)motivazione } \\
\text { (I)passione } \\
\text { (I)poco_impegno- } \\
\text { (I)scarsa autorevolezza } \\
\text { (I)simpatia } \\
\text { (I)tolleranza } \\
\text { (S)troppe_vacanze- }\end{array}$ & $\begin{array}{l}6 \\
7 \\
7 \\
8 \\
5 \\
8 \\
9 \\
6 \\
7 \\
8 \\
7 \\
7 \\
8 \\
9 \\
8 \\
6 \\
7 \\
8 \\
6\end{array}$ & $\begin{array}{l}2.833 \\
3.286 \\
3.174 \\
2.750 \\
3.200 \\
3.000 \\
3.444 \\
2.833 \\
2.714 \\
2.500 \\
2.857 \\
3.143 \\
2.625 \\
3.556 \\
2.500 \\
3.176 \\
3.143 \\
3.125 \\
2.500\end{array}$ \\
\hline
\end{tabular}

Nel caso del "percepito" non si è ritenuto opportuno chiedere di indicare le evocazioni per ordine di importanza in quanto esse si riferiscono a idee di terze persone e quindi, a nostro parere, difficilmente organizzabili in priorità se non attraverso una soggettività del "probabile" molto diversa dalla scelta personale consapevole dei due casi precedenti. Per questo motivo non è stato svolto il test di centralità per queste evocazioni.

Come è possibile rilevare dalla Tabella 3 , il nucleo centrale della probabile rappresentazione professionale dell'insegnante percepito risulta essere abbastanza simile a quella reale. Ritroviamo anche qui cinque caratteristiche presenti nei nuclei centrali delle altre due rappresentazioni: competenza, competenza materia, pazienza, preparazione e professionalità. Adattabilità e flessibilità, presenti nel precedente nucleo centrale, sono qui sostituite da disponibilità mentre le caratteristiche negative aumentano in presenza e in "significanza": non più segnali di semplice fatica ma veri e propri segnali negativi legati alla professione (impreparazione, incompetenza e scarsa professionalità) e al confronto sociale (lavorano poco, fannullone e privilegiati). Riappare inaspettatamente una componente relazionale, comprensione, che in precedenza non appariva nel nucleo centrale, benché tale componente possa, a nostro avviso, essere intesa anche come tratto individualistico "umano" oltre che come competenza relazionale vera e propria.

Nel procedere alla creazione di categorie di prossimità semantica qui ne sono state create 31 , che includono 950 evocazioni sul totale iniziale di $1445(65.7 \%)$, contro le 27 del "reale" (69.8\% di evocazioni incluse) e le 29 dell'ideale ( $83.4 \%$ ), segno di una condivisione delle caratteristiche inferiore rispetto alle altre due tipologie di insegnante. 

Grafico 3 - Rappresentazione dei legami tra categorie delle evocazione (albero massimo) per
insegnante percepito

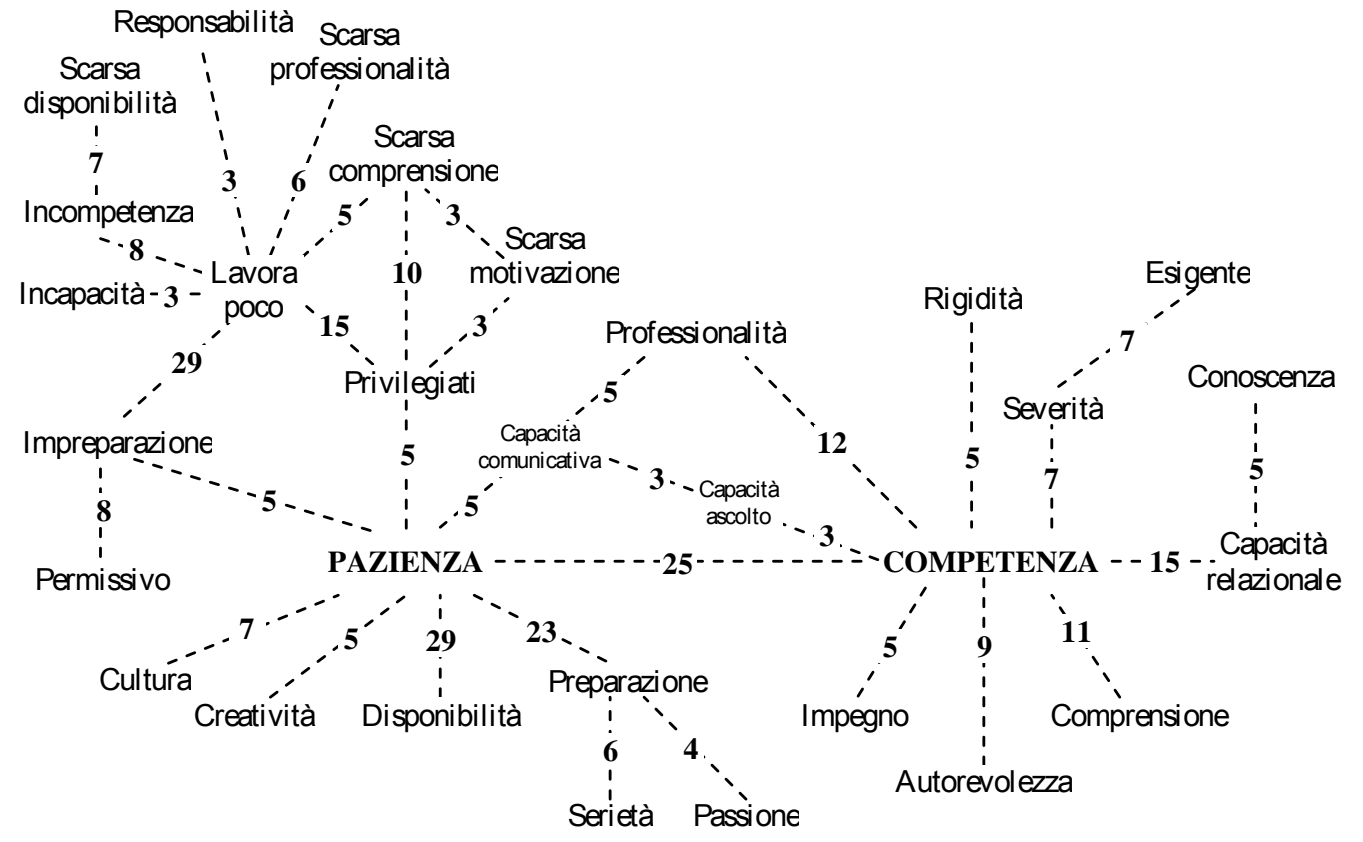

Anche i legami tra le caratteristiche dell'insegnante "percepito" confermano la similarità di questa rappresentazione con quella dell'insegnante "reale" (si veda il Grafico 3). Infatti ritroviamo gli stessi due principi organizzatori, pazienza e competenza, attorno ai quali si collegano tutti gli altri. Salta subito agli occhi come, in questo caso, le relazioni siano molto più numerose con pazienza, caratteristica individualepersonale, rispetto a competenza.

Ritroviamo nuovamente la capacità relazionale collegata a competenza, confermando quindi, a nostro avviso, come la competenza "tecnica" non possa fare a meno della sua specificità relazionale ed una sua declinazione umana (comprensione). La pazienza, così come una certa dose di preparazione e di disponibilità, vengono percepite come doti centrali, riconosciute anche dagli "altri". Inoltre, accanto a caratteristiche positive (creatività, cultura, capacità comunicativa) ritroviamo un gruppo

\section{Confronti tra nuclei centrali}

Prendiamo in considerazione i primi quadranti delle tre "tipologie". che rappresentano il probabile nucleo centrale di ciascuna rappresentazione dell'insegnante:

\begin{tabular}{l|l|l}
\hline \multicolumn{1}{c|}{ IDEALE } & \multicolumn{1}{|c}{ REALE } & \multicolumn{1}{c}{ PERCEPITO } \\
\hline & & (P)competenza \\
& & (P)competenza_materia \\
& (I)adattabilità & (P)comprensione \\
& (P)competenza & (I)disponibilità \\
(I)autorevolezza & (P)competenza_materia & (I)esigente \\
(R)capacità_relazionale & (P)competenza_didattica & (S)fannullone- \\
(P)competenza & (P)conoscenza_materia & (P)impreparazione- \\
(P)competenza_materia & (I)demotivazione- & (P)incompetenza- \\
(I)empatia & (I)impegnato & (S)lavora poco- \\
(I)motivazione & (I)flessibilità & (I)pazienza \\
(I)passione & (I)pazienza & (I)permissivo \\
(I)pazienza & (I)passione & (P)preparazione \\
(P)preparazione & (S)privilegiati- \\
(P)professionalità & (P)proparazione & (P)professionalità \\
& (I)stanchezza & (I)rigidità- \\
& (I)stressato- & (I)scarsa motivazione- \\
& & (P)scarsa professionalità- \\
& & (I)tuttologo \\
\hline
\end{tabular}
piuttosto consistente di caratteristiche negative sia professionali, sia individuali, sia sociali, segno di una presenza, per la verità attesa, non trascurabile di stereotipi negativi, veicolati anche dai media, nella rappresentazione della percezione della classe docente.

Come si può notare, tutti e tre i nuclei centrali presentano cinque evocazioni costanti: competenza, competenza materia, pazienza, preparazione e professionalità. Questa costante suggerisce una conferma del ruolo centrale delle caratteristiche professionali della rappresentazione dell'insegnante, sia esso idealmente concepito, realmente osservato o presumibilmente percepito da altri. Piuttosto interessante è invece la scomparsa delle caratteristiche relazionali dal nucleo centrale 
dell'insegnante "reale", interpretabile come se gli insegnanti stessi riconoscessero nella propria categoria una qualche carenza dal punto di vista delle capacità comunicative-relazionali, caratteristiche che viceversa risultano centrali nell'insegnante ideale. Degna di nota è anche "l'irruzione" nel nucleo centrale di caratteristiche negative: se era prevedibile aspettarsi un risultato del genere riguardo all'insegnante percepito, non altrettanto prevedibile lo è la comparsa di caratteristiche negative nell'insegnante reale. Inoltre le caratteristiche "negative" della rappresentazione "reale" sono riferibili in qualche misura alla difficoltà individuale del ruolo (demotivazione, stanchezza, insoddisfazione, sfiducia, stress), mentre nel "percepito" sono, se non prevalenti, almeno molto presenti caratteristiche negative di tipo sociale (lavora poco, privilegiato, fannullone) e tecnicoprofessionale (impreparazione, incompetenza, scarsa professionalità). Infine, come ci si poteva attendere, le caratteristiche "sociali" compaiono solo nel nucleo del "percepito". Per quanto riguarda i due quadranti delle caratteristiche "intermedie" (alta frequenza di evocazione e basso rango e bassa frequenza di evocazione e alto rango) una prima considerazione riguarda le caratteristiche personali, numericamente molto più consistenti rispetto alle altre categorie di evocazioni, nell'insegnante "ideale" e nel "reale" ma non nel "percepito": in quest'ultimo risaltano invece maggiormente le caratteristiche tecnico-professionali. sia in positivo che in negativo. È da notare, inoltre, che la motivazione, presente nel nucleo centrale della rappresentazione "ideale", nelle altre due rappresentazioni viene "relegata" nell'ultimo quadrante, riferibile alle rappresentazioni individuali o di piccoli gruppi (ABRIC, 1994).

\section{Considerazioni conclusive}

Lo scopo del presente studio era quello di indagare se esistesse o meno una differenza, una disarmonia nella rappresentazione dell'identità professionale, del proprio Sé professionale, diviso tra ideale e reale e percepito oltre a cercare evidenze a supporto dell'ipotesi formulata da Lodolo D'Oria ovvero se questa disarmonia possa essere fonte di innesco di stati emozionali negativi, di sofferenza, disagio, burnout o viceversa se l'armonia tra le due tipologie del Sé (ideale e reale) e l'equilibrio tra identità personale e sociale possano promuovere il benessere.

L'analisi delle caratteristiche evocate dai partecipanti è stata effettuata utilizzando il software EVOCATION 2000 il quale ha permesso di identificare i rispettivi nuclei centrali in cui venivano presentate le caratteristiche evocate con più frequenza e per prime (basso rango). II confronto tra le caratteristiche che compongono i tre differenti nuclei che, lo ricordiamo, forniscono attendibili indicazioni circa la probabile "struttura" portante della rappresentazione dell'identità professionale degli insegnanti, rivelano alcune costanze: competenza generale, competenza della materia, pazienza, preparazione e professionalità sono presenti in tutti e tre i "nuclei". Ciò suggerisce che le caratteristiche professionali giochino un ruolo di rilievo nella rappresentazione dell'insegnante, sia esso idealmente concepito, realmente osservato o presumibilmente percepito da altri. Più interessante è, a nostro giudizio, la scomparsa delle caratteristiche relazionali dal nucleo centrale dell'insegnante "reale", probabile segno di riconoscimento nella propria categoria di una qualche carenza dal punto di vista delle capacità comunicativerelazionali, caratteristiche risultate invece centrali nell'insegnante ideale. Degna di nota è anche la comparsa nel nucleo centrale dell'insegnante reale di caratteristiche negative, riferibili in qualche misura alla difficoltà individuale del ruolo (demotivazione, stanchezza, insoddisfazione, sfiducia, stress). Anche nel nucleo centrale del "percepito" sono molto presenti caratteristiche negative, ma esse sono di tipo sociale (lavora poco, privilegiato, fannullone) e tecnico-professionale (impreparazione, incompetenza, scarsa professionalità), interpretabili come una relativamente diffusa insicurezza del ruolo (pressione e responsabilità sociale) e/o una parziale conferma dello scarso prestigio sociale che oggi l'insegnate si trova a subire. Per quanto riguarda invece le caratteristiche "intermedie" (alta frequenza di evocazione ma basso rango o bassa frequenza di evocazione ma alto rango) si è rilevato che le caratteristiche personali sono più numerose rispetto alle altre categorie di evocazioni nell'insegnante "ideale" e nel "reale" ma non nel "percepito": in quest'ultimo risaltano invece maggiormente le caratteristiche tecnico-professionali, sia in positivo che in negativo. Infine la motivazione, presente nel nucleo centrale della rappresentazione "ideale", nelle altre due rappresentazioni viene relegata nell'ultimo quadrante, riferibile alle rappresentazioni individuali o di piccoli gruppi (ABRIC, 1998).

Riteniamo si possa affermare che gli insegnanti possiedano una rappresentazione della propria identità professionale abbastanza "armonica": l'esistenza di caratteristiche (professionali) costanti nei nuclei centrali delle tre diverse "identità" proposte ci spingono a trarre questa conclusione. Tuttavia riteniamo importante evidenziare almeno tre assi di "disarmonia" rilevabili: il primo riguarda l'importanza delle competenze relazionali, ritenute idealmente centrali ma non altrettanto presenti nella quotidianità (insegnante reale); il secondo evidenzia segnali di fatica, di demotivazione che fanno parte 
dell'immagine professionale che gli insegnanti hanno della propria categoria e che trova ulteriore sostegno anche nella rappresentazione "percepita"; il terzo, forse il più significativo, riguarda il binomio competenza-relazionalità attorno a cui si legano le altre caratteristiche possedute dall'insegnante ideale, binomio che si trasforma in personalità-competenza nelle altre due rappresentazioni. È questa, in ultima analisi, la discrepanza più rilevante che ci sembra esistere tra le diverse "identità": le caratteristiche professionali sono considerate una costante identitaria mentre la competenza relazionale non rientra a pieno titolo tra le caratteristiche centrali realmente possedute dagli insegnanti.

\section{Referências}

ABRIC, J. C. L'organisation interne des representations sociales: système central et système périphérique. In: GUIMELLI, C. (Ed.). Structures et transformations des représentations socials. Lausanne: Delachaux et Niestlé, 1994. p. 73-84.

. A abordagem estrutural das representações sociais. In: MOREIRA, A. S. P.; OLIVEIRA, D. C. (Orgs.). Estudos interdisciplinares de representação social. Goiânia: AB, 1998. p. 27-38.

BERGER, P. L.; LUCKMANN, T. La realtà come costruzione sociale. Bologna: II Mulino, 1969.

BROWN, R. Psicologia sociale dei gruppi. Bologna: II Mulino, 2000.

CARUGATI, F. II Sé e I'Identità: alla ricerca di una nuova teoria. Faenza: Stabilimento Grafico F.lli Lega, 1979.

CHIOSSO, G.; MARIANI, A. M. Teorie della scuola e professione docente. In: ARACE et al. La formazione degli insegnanti: scienze dell'educazione e nuova professionalità docente. Torino: UTET, 2002.

CORRADINI, L. Sociologia dell'educazione e dimensione antropologico-etica della professione docente. In: PETRINI, E. (Eds.). Nuove prospettive della professione docente. Roma: Istituto della Enciclopedia Italiana, 1982.

DURKHEIM E. La sociologia e l'educazione. Milano: Newton Compton, 1971.

ERICKSON, E. Gioventù e crisi d'identità. Roma: Armando, 1974.

FLAMENT, C. Aspects périphériques des representations sociales. In: GUIMELLI, C. (Ed.). Structures et transformations des représentations sociales. Lausanne: Delachaux et Niestlé, 1994. p. 85118.

HARGREAVES, A. Changing teachers, changing times: teachers' work and culture in the postmodern age. London: Cassel, 1994.
HUBERMAN, A. M.; VANDENBERGHE, R. (Eds.). Understanding and Preventing Teacher Burnout: a sourcebook of international research and practice. Cambridge: Cambridge University Press, 1999.

JODELET, D. Representation Sociales: phenomenes, concepts et theorie. In: MOSCOVICl, S. (Eds.). Psychologie sociale. Paris: PUF, 1984.

KELCHTERMANS, G. Teaching career: between burnout and fading away? Reflection from a narrative and biographical perspective. In: VANDENBERGHE, R.; HUBERMAN, A. M. (Eds.). Understanding and Preventing Teacher Burnout: a sourcebook of international research and practice. Cambridge: Cambridge University Press, 1999. p. 176-191.

KYRIACOU, C. Teacher stress and burnout: an international review. Educational Research, n. 29, p. 146-152, 1987.

LISIMBERTI, C. L'identità professionale come progetto. Milano: Vita e Pensiero, 2006.

LODOLO D'ORIA, V.; PECORI GIRALDI, F.; VITELLO, A.; VANOLI, C.; ZEPPEGNO, P.; FRIGOLO, P. Burnout e patologie psichiatriche degli insegnanti, 2002. Disponível em: <http://www.edscuola.it/archivio/ psicologia/burnout.pdf>. Acesso em: 11 apr. 2011.

LODOLO D'ORIA, V.; POCATERRA, R.; POZZI, S. La percezione el burnout negli insegnanti: risultati di una ricerca nazionale condotta su 1252 insegnanti, 2003. Disponível em: <http://www.edscuola.it/archivio/ interlinea/burnout_00.pdf>. Acesso em: 11 apr. 2011.

MIUR. Università e Ricerca. Ministero Dell'Istruzione. Indicazioni per il curricolo. Roma: Miur, 2007.

MOLINER, P. Les metodes de repaerage et d'identification des representations sociales. In: GUIMELLI, C. (Org.). Structures et transformations des representations sociales. Lausanne: Delanchaux \& Niestlè, 1994. p. 199-232.

MOSCOVICl, S. Attitudes and opinions. Annual Review of Psychology, n. 7, p. 231-260, 1963.

. La psychanalyse, son image et son public. Paris: PUF, 1961/1976.

NIAS, J. Changing times, changing identities: grieving for a lost self. In: BURGESS, R. (Ed.). Educational research and evaluation policy and practice. London: Falmer, 1993.

OCSE. Italian contribution to attracting, developing and retaining effective teachers. Paris: OECD, 2003.

Teachers Matter: attracting, developing and

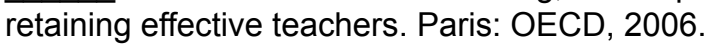

PALMONARI, A. Psicologia. Bologna: II Mulino, 1981.

PALMONARI, A.; CAVAZZA, C.; RUBINI, M. Psicologia sociale. Bologna: II Mulino, 2002.

PORCHEDDU, A. Gli insegnanti: un ruolo sociale in trasformazione. In: BROCCOLI, A. Ruolo, status e 
formazione dell'insegnante italiano dall'Unità a oggi. Milano: ISEDI, 1978. p. 133-211.

SANTINELLO, M.; NEGRISOLO, A. Quando ogni passione è spenta: la sindrome del burnout nelle professioni sanitarie. Milano: Mc Graw-Hill, 2009.

TAJFEL, H. Gruppi umani e categorie sociali. Bologna: II Mulino, 1995.

VERGÉRS, P. L'Evocation de l'argent: Une méthode pour la definition du noyage central d'une representation. Bulletin de Psychologie, Tome XLV, n. 405, p. 204209, 1992.

Ricevuto: 16/06/2011

Accettato: 20/07/2011 